\title{
Nanocontainers in and onto Nanofibers
}

\author{
Shuai Jiang, Li-Ping Lv, Katharina Landfester, * Daniel Crespy*
}

\section{Miniemulsion for the formation of nanocontainers}

Miniemulsion droplets are stabilized against coalescence by surfactants while Ostwald ripening is hindered by the addition of a so-called hydrophobe or lipophobe in oil-in-water or water-in-oil systems, respectively. Each droplet can be considered as an independent nanoreactor, allowing the design of complex structures with sophisticated materials, such as nanocapsules with core-shell structure.

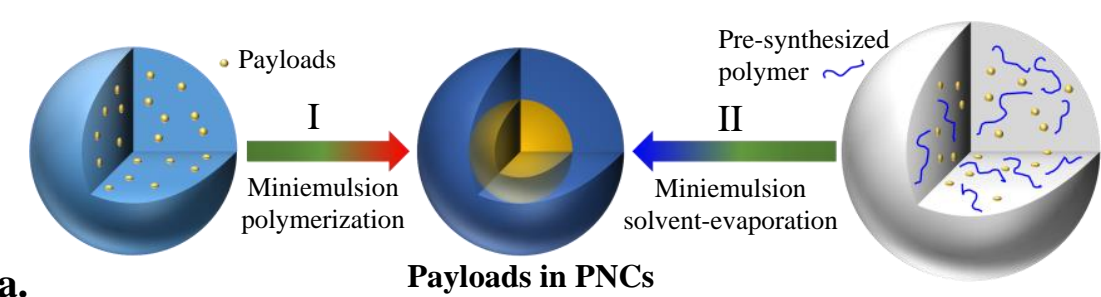

b.

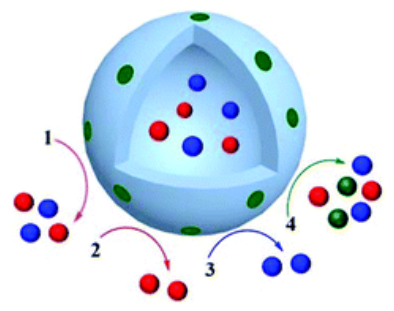

Figure S1. a: Scheme showing the possible pathways for the formation of polymer nanocapsules in miniemulsion that are miniemulsion polymerization (I) and miniemulsionsolvent evaporation (II). ${ }^{1}$ b: Nanocapsules with programed logical gates for selective or nonselective release of payloads. Reproduced with permission from ref. 1. Copyright 2015 The Royal Society of Chemistry.

\section{Formation of lipid-based colloids}

Vesicles could be transformed to liquid crystalline nanoparticles upon evaporation of ethanol. Hexagonal lattices, lined and coiled structures, and disordered structures were created and controlled by the composition of a mixture of surfactant, water, ethanol, and phytantriol. ${ }^{2}$ Bovine serum albumin and cytochrome, two water-soluble proteins, were loaded in water-in- 
oil microemulsions. ${ }^{3}$ The microemulsion was then dispersed in water to yield particles with micellar cubic structures. The release of cytochrome was found to be dependent on the internal structure of the particles. It was faster from the micellar cubosomes than from the emulsified microemulsion. $^{3}$

\section{References}

(1) Zhao, Y.; Lv, L.-P.; Jiang, S.; Landfester, K.; Crespy, D. Advanced Stimuli-Responsive Polymer Nanocapsules with Enhanced Capabilities for Payloads Delivery. Polym. Chem. 2015, 6, 4197-4205.

(2) Kim, D.H.; Lim., S.; Shim, J.; Song, J.E.; Chang, J.S.; Jin, K.S.; Cho, E.C. A Simple Evaporation Method for Large-Scale Production of Liquid Crystalline Lipid Nanoparticles with Various Internal Structures. ACS Appl. Mater. Interf. 2015, 7, 20438-20446.

(3) Chemelli, A.; Maurer, M.; Geier, R.; Glatter, O. Optimized Loading and Sustained Release of Hydrophilic Proteins from Internally Nanostructured Particles. Langmuir 2012, 28, 16788-16797. 